\title{
On the ionizing source of the Flame Nebula (NGC 2024)
}

\author{
Arjan Bik, Annique Lenorzer, Lex Kaper, Alex de Koter, Rens Waters \\ Sterrenkundig Instituut Anton Pannekoek, Universiteit van Amsterdam, \\ Kruislaan 403, NL-1098 SJ Amsterdam, Nederland
}

Fernando Comerón

European Southern Observatory, Karl-Schwarzschild-Straße 2, Garching-bei München, D-85748, B.R.D.

\author{
Margaret M. Hanson \\ Department of Physics, University of Cincinnati, \\ Cincinnati, OH 45221-0011, USA
}

\begin{abstract}
We have identified IRS $2 \mathrm{~b}$ as the ionizing source of the Flame Nebula (NGC 2024). For several decades it has been clear that such a hot, massive star must be present in this heavily obscured region, and now it has been found. New $J$ - and $K$-band photometry and a VLT-ISAAC $K$-band spectrum show that IRS $2 \mathrm{~b}$ is an $08-09.7 \mathrm{~V}$ star, consistent with published radio continuum and recombination line observations. IRS $2 \mathrm{~b}$ seems to be slightly evolved from the zero-age main sequence.
\end{abstract}

\section{Introduction}

The Orion complex hosts several well-known $\mathrm{H}$ II regions, such as the famous Orion Nebula and the compact H II region NGC 2024. In the optical, NGC 2024 appears as a bright nebula straddled by a north-south oriented dust lane, which obscures the central part of the nebula. This has made it very difficult to identify the main ionizing source of this $\mathrm{H}$ II region. Obviously, infrared imaging and spectroscopy are needed to obtain a full inventory of the stars in the central region of NGC 2024. Such studies have provided several candidates for the ionizing source of NGC 2024 (e.g., NGC 2024-IRS2, Grasdalen 1974), but none of these candidates are very convincing.

\section{The nature of the ionizing source}

We present three independent diagnostics that help to constrain the position of IRS 2b in the Hertzsprung-Russell diagram (cf. Bik et al. 2002). The derived temperature and luminosity of IRS $2 b$ indicate that it is the likely ionizing source of NGC 2024. The three arguments we can offer are: $(i)$ the $J$ - and $K$ magnitude; (ii) the $K$-band spectrum; and (iii) the radio continuum flux of NGC 2024. 

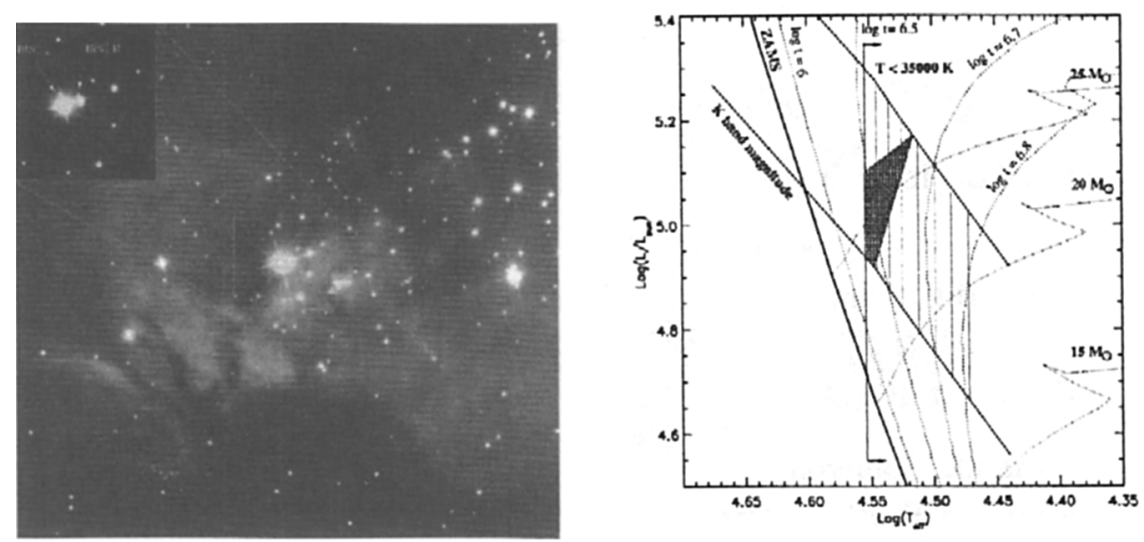

Figure 1. Left: Near-infrared image of NGC $2024\left(\mathrm{~Pa} \beta, \mathrm{H}_{2}, \mathrm{Br} \gamma\right)$. The insert shows IRS $2 \mathrm{~b}$, the ionizing source of the Flame Nebula. Right: H-R diagram, including the derived constraints on $L$ and $T_{\text {eff }}$ of IRS $2 \mathrm{~b}$.

Adopting a distance $d=363 \mathrm{pc}$ (Brown et al. 1994) and a reddening of $V=24 \mathrm{mag}$ (using an anomalous extinction law with $R_{V}=5.5$, Lee 1968), the $K$-band magnitude can be converted into luminosity for a given temperature, thus constraining its location in the H-R diagram (Figure 1 ). The $K$-band spectrum of IRS $2 \mathrm{~b}$ includes photospheric absorption lines of HeI $(2.1128,2.1137 \mu \mathrm{m})$ and $\operatorname{Br} \gamma(2.1661 \mu \mathrm{m})$, indicating that its spectral type is cooler than $\mathrm{O} 7.5 \mathrm{~V}$, corresponding to $T_{\text {eff }}<35000 \mathrm{~K}$ (Hanson et al. 1996).

The number of ionizing continuum photons derived from radio continuum maps (e.g., Barnes et al. 1989) compared with stellar models of Smith et al. (2002) provides a further constraint in the H-R diagram (Figure 1). The resulting range in $T_{\text {eff }}(32500$ to $35000 \mathrm{~K})$ and in $\log \left(L / L_{\odot}\right)(4.8$ to 5.2$)$ is consistent with a spectral type O8-09.7 V (Martins et al. 2002). Its location in the H-R diagram suggests that IRS $2 \mathrm{~b}$ is slightly evolved from the ZAMS, close to the isochrone of $3 \times 10^{6} \mathrm{yr}$.

\section{References}

Barnes, P.J., Crutcher, R.M., Bieging, J.H., et al. 1989, ApJ 342, 883

Bik, A., Lenorzer, A., Kaper, L., et al. 2002, A\&A submitted

Brown, A.G.A., de Geus, E.J., de Zeeuw, P.T. 1994, A\&A 289, 101

Grasdalen, G.L. 1974, ApJ 193, 373

Hanson, M.M., Conti, P.S., Rieke, M.J. 1996, ApJS 107, 281

Lee, T.A. 1968, ApJ 152, 913

Martins, F., Schaerer, D., Hillier, D.J. 2002, A\&A 382, 999

Smith, L.J., Norris, R.P.F., Crowther, P. 2002, MNRAS 337, 1309 